\title{
Metamaterial Analog of Electromagnetically Induced Transparency
}

\author{
N. Papasimakis, ${ }^{*}$ V. A. Fedotov, and N. I. Zheludev \\ Optoelectronics Research Centre, University of Southampton, SO17 1BJ, United Kingdom
}

\section{S. L. Prosvirnin}

Institute of Radio Astronomy, National Academy of Sciences of Ukraine, Kharkov, 61002, Ukraine (Received 6 January 2008; revised manuscript received 12 November 2008; published 19 December 2008)

\begin{abstract}
We demonstrate a classical analog of electromagnetically induced transparency in a planar metamaterial. We show that pulses propagating through such metamaterials experience considerable delay. The thickness of the structure along the direction of wave propagation is much smaller than the wavelength, which allows successive stacking of multiple metamaterial slabs leading to increased transmission and bandwidth.
\end{abstract}

Changes in the velocity of light propagating through dispersive media have been the subject of extensive investigations in the past [1]. It was predicted [2] and later observed $[3,4]$ that light pulses can propagate with apparent velocities greater or smaller than in vacuum without strong distortion due to pulse reshaping phenomena. Today, control over these effects is essential for the development of optical communication technologies leading to a number of different approaches [5-16], such as the nonlinear quantum phenomenon of electromagnetically induced transparency (EIT) [17,18]. In that case, an opaque atomic medium is rendered transparent in a narrow spectral region within the absorption line through quantum interference of a pump and a probe laser beam tuned at different transitions [17]. This has important consequences such as dramatically reducing the group velocity [5] and enhancing nonlinear interactions [19].

Most of the proposed techniques, however, involve special and often cumbersome experimental conditions, such as cryogenic temperatures and high intensity lasers. Recently, the implementation of EIT-like behavior in linear classical systems has attracted a lot of attention [20-28], since in this case the operating frequency can be tuned by varying the system geometry, and no pumping is necessary. Interference between coupled classical resonators can lead to EIT-like effects, i.e., narrow transmission resonances within the single-resonator stop-band. Nevertheless, all existing solutions have fundamental constraints on the minimum thickness of the medium along the propagation direction and implementing slow-light effects in compact configurations remains challenging. However, considerable pulse delays have recently been reported in planar metamaterials (also known as frequency selective surfaces or metafilms), namely, metal-dielectric slabs of vanishing thickness along the propagation direction, periodically patterned on a subwavelength scale [29]. It has been suggested for the first time that electromagnetic behavior of planar metamaterials may represent a classical analog of
EIT [30]. In this letter, we present a clear demonstration of the intriguing analogy and discuss its origin in detail. We show that the phenomenon arises as a result of engaging "trapped-mode" resonances [31-33] that are weakly coupled to the free space. Intriguingly, such resonant modes appear to be analogous to the narrow resonances observed in electromagnetically induced transparency [33]. Finally, we demonstrate significant bandwidth enhancement in cascaded metamaterials, while the resulting structure is still of subwavelength thickness, thus paving the way to the realization of extremely compact optical components with high integration efficiency.

The studied metamaterials are based on the continuous fish-scale metallic pattern [34]. To illustrate the difference between a typical single band-gap resonant structure and the proposed "trapped-mode" metamaterial, we investigated two types of structures. In the reference structure, the metallic pattern is etched on one side of the dielectric slab, while in the "trapped-mode" metamaterial the pattern resides on both sides of the PCB laminate, so that the pattern on one side of the dielectric slab is shifted along the meandering strips by half a translational cell with respect to the pattern on the other side (see Fig. 1).

The transmission and reflection properties of the metamaterials were measured in an anechoic chamber at normal incidence, in the frequency range of 2 to $14 \mathrm{GHz}$, using two broadband horn antennas and a vector network analyzer. The measured spectra for the single- and double-layer fish scales are presented in Figs. 2(a) and 2(b), respectively. In the reference case of the single fish-scale (SFS) structure, a wide stop band, typical of FSS, is visible, centered at 6.5 $\mathrm{GHz}$ and accompanied by anomalous dispersion (negative phase derivative). On the contrary, when the double-layered fish-scale (DFS) metamaterial is considered [Fig. 2(b)], the spectrum exhibits a narrow transmission resonance centered at around $5.5 \mathrm{GHz}$, in the middle of the stop band of the reference structure. The width of the transparency window is $0.5 \mathrm{GHz}$, while transmission 


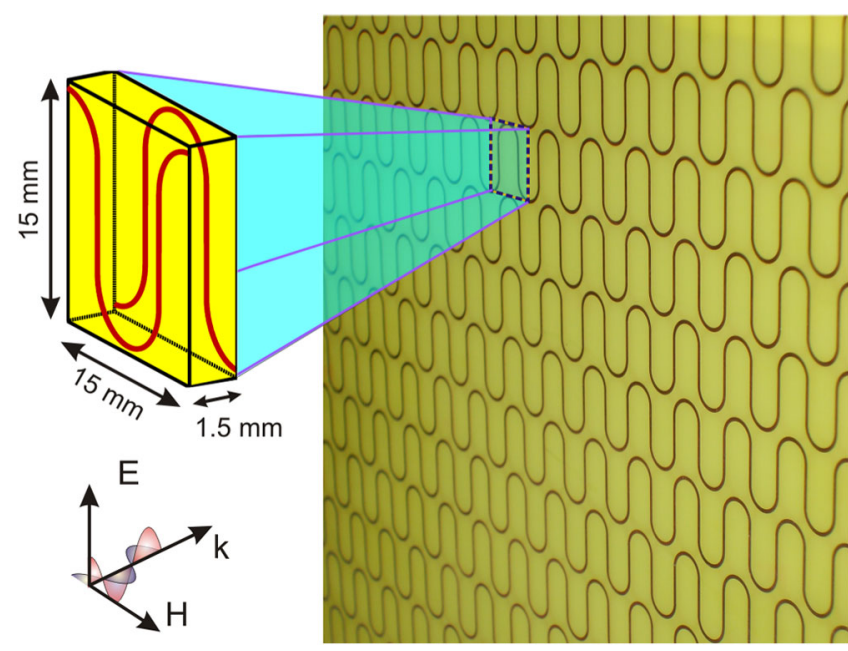

FIG. 1 (color online). The bilayered fish-scale metamaterial and its unit cell. The size of the translational cell was $15 \mathrm{~mm} \times$ $15 \mathrm{~mm}$ rendering the metamaterials nondiffracting at frequencies below $20 \mathrm{GHz}$. The two fish-scale patterns (red) are etched in the top and bottom face of a $1.5 \mathrm{~mm}$ thick dielectric, respectively, and are displaced along the meandering stripes by a half unit cell in respect to one another.

levels exceed $20 \%$. As a result, very sharp normal dispersion (positive phase derivative) is also observed $\left(\sim 350^{\circ} / \mathrm{GHz}\right)$, which can lead, subsequently, to long pulse delays.

The dispersive properties of the bilayered fish-scale metamaterial can be further illustrated by considering propagating electromagnetic pulses. To this end, we reconstruct the response of the metamaterial by applying the inverse Fourier transform to the convolution of the transmission spectrum with the pulse power spectrum. In particular, we consider a 2.5 ns long Gaussian-shaped pulse centered at the trapped-mode resonance, propagating through the DFS array. The pulse will be delayed by $\sim 1 \mathrm{~ns}$, greater than $1 / 3$ of the incident pulse width [see Fig. 2(c)] which is remarkable in view of the vanishing thickness of the structure $(\approx \lambda / 35)$. It essentially retains its Gaussian shape with the exception of a weak broadening, since most of its spectral power lies in regions of constant phase derivative. Moreover, the transmission is reasonably high, exceeding $\sim 15 \%$ (may be improved by using substrates with lower losses), and enables the successive stacking of metamaterial slabs.

The observed response of the DFS metamaterial is a direct classical analog of EIT, as the weak coupling of the counter-propagating currents to free space in the metamaterial is reminiscent of the weak probability for photon absorption in EIT observed in atomic systems [17]. The main difference lies in the fact that, in the present case, the transparent state is a result of classical field interference, rather than quantum interference of atomic excitation pathways. To draw the parallel further, the response of an atomic system to an incident field $E_{i}$ can be described by the induced polarization $P=\chi E_{i}$, where absorption is
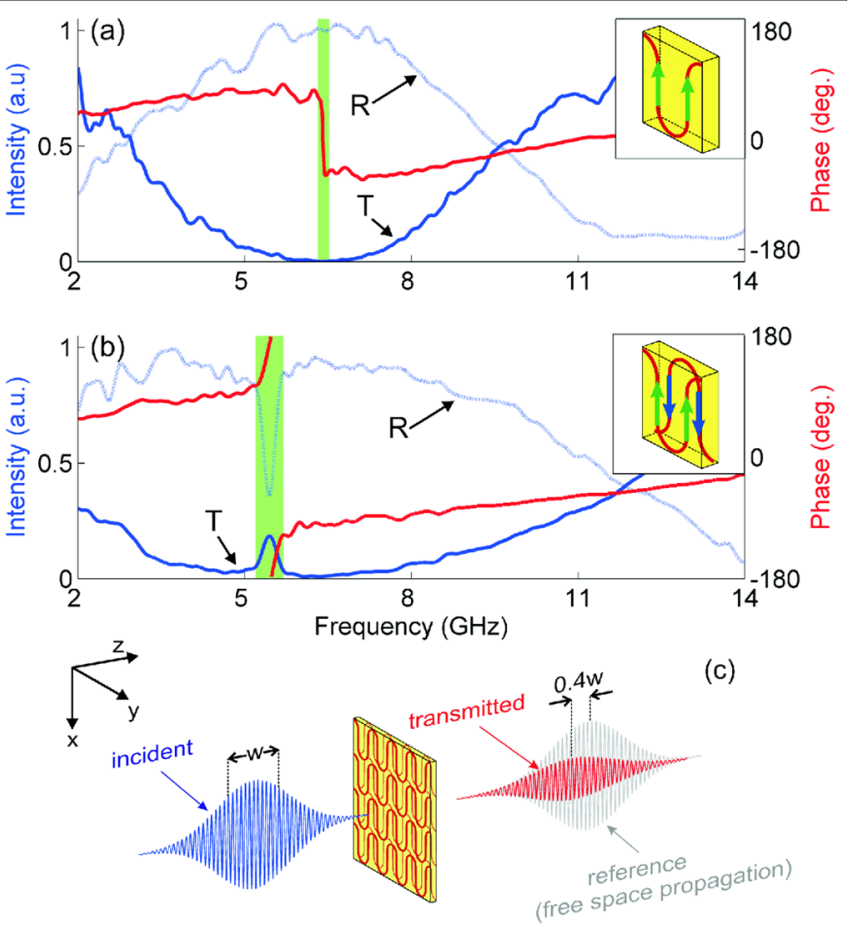

FIG. 2 (color online). Experimentally measured reflection amplitude (dotted blue), transmission amplitude (blue) and transmission phase change (red) of the single (a) and the bilayered (b) fish-scale metamaterial. Frequency regions of resonant anomalous (a) and normal (b) dispersion are highlighted. In both cases the polarization is along the vertical axis (inset to Fig. 1). (c) Reconstructed response of the bilayered fish-scale (red) to a $2.5 \mathrm{~ns}$ microwave Gaussian pulse (blue) with center frequency at the maximum of the transmission window. The transmitted pulse is delayed by $40 \%$ of the pulse width, when compared to a pulse propagating through free space (gray).

proportional to the imaginary part of the linear susceptibility $\chi$. Similarly, the metamaterial response could be expressed through the induced currents $i_{1}, i_{2}$ on the two layers. Here the relation between the incident field and the induced currents can be derived by following an equivalent circuit approach [31]. The response of each layer of the metamaterial can be described by a combination of the unit cell impedance $Z_{0}=Z_{s}+Z_{m}$ (which includes both the unit cell element self impedance $Z_{s}$ and the mutual impedance between different elements on the same layer $Z_{m}$ ) and the impedance $Z_{c}$, which accounts for the interlayer coupling and the inversion of the second layer with respect to the first one. Near the unit cell element resonance $\omega_{o}$, only the term $Z_{0}$ varies rapidly with frequency and can be approximated by: $Z_{0}=R_{s}+Z_{m}+j L_{s}\left(\omega^{2}-\omega_{o}^{2}\right) / \omega \simeq$ $\left(R_{s}+Z_{m}\right)+2 j L_{s}\left(\omega-\omega_{o}\right)$, where $R_{s}$ and $L_{s}$ can be assumed constants defined by the geometry of the structure. The induced currents, $i_{1}$ and $i_{2}$, on the front and back metamaterial layers, respectively, will be excited with a phase difference $\phi$ due to their subwavelength physical separation. These currents will be proportional to the incident field $E_{i}$ and for later convenience can be written as: 
$i_{1} \sim e^{i \phi} E_{i} Z_{1} / D$ and $i_{2} \sim E_{i} Z_{2} / D$, where $Z_{1}=Z_{0} e^{-i \phi}-$ $Z_{c}, Z_{2}=Z_{0} e^{i \phi}-Z_{c}, D=Z_{0}^{2}-Z_{c}^{2}$. Neglecting dissipation effects, the losses in the metamaterial will be given by the reflection coefficient $|R|$ :

$$
|R| \sim\left|i_{1}+i_{2} e^{i \phi}\right| \sim\left|\frac{Z_{1}+Z_{2}}{Z_{1} Z_{2}+Z_{c}\left(Z_{1}+Z_{2}\right)}\right| .
$$

Equation (1) contains the distinctive features observed in induced transparency systems and is very similar to the absorption relation in the dressed-state picture of EIT. More concisely, in contrast to the bare state picture, where both the pump and the probe beams have to be considered, here the effect of the pump is translated to a splitting of the resonance, at which the probe beam is tuned leading to two dressed states with closely spaced resonant frequencies. Between these resonances, the contributions of the dressed states interfere destructively leading to an absorption minimum $[35,36]$. This has a direct analogy with the DFS, where it is actually the physical separation of the layers that induces the resonance splitting, resulting in two separate modes, each corresponding to strong dipolelike current oscillations on either the first or second metamaterial layer. In a narrow frequency region, these modes coexist with opposite phases, which leads to an antisymmetric current configuration. In the far-field zone, the waves emitted by this current configuration interfere destructively, hence dramatically reducing scattering losses (reflection) and facilitating transmission. In terms of impedance, the transparency regime corresponds to a minimum of $Z_{1}+Z_{2}$ in Eq. (1) (i.e., when two layers are excited with opposite phases). This is illustrated for a characteristic case of Eq. (1) in Fig. 3(a), where the EIT signature of a very narrow transparency inside the stop band can be seen. Since the radiation losses are at a minimum, the currents induced in the metal stripes in each layer become exceptionally high leading to strong concentration of energy between the layers. This is illustrated in Figs. 3(b) and 3(c) by finite element modeling of the bilayered fish-scale structure using a commercial solver (COMSOL 3.4), where the antisymmetric current configuration and the electromagnetic energy density at the trappedmode resonance are presented. This resonance is not accessible for $\phi=0$ (perfectly overlapping layers), since in this case $Z_{1}=Z_{2}$ and Eq. (1) reduces merely to $|R| \sim$ $\left(Z_{0}+Z_{c}\right)^{-1}$.

Finally, we show that successive stacking of metamaterial layers can surpass the bandwidth restrictions inherent to resonant phenomena. Although multilayered structures have been employed to create broadband filters [32], here we aim to create a wide band of sharp normal dispersion with constant phase derivative. The fish-scale design can be cascaded in such a way, that each slab is inverted with respect to the adjacent ones (see insets to Fig. 4), while the distance between successive patterns is defined by the thickness of the substrates $(1.5 \mathrm{~mm})$. The experimental results for three and four fish-scale layers are shown in

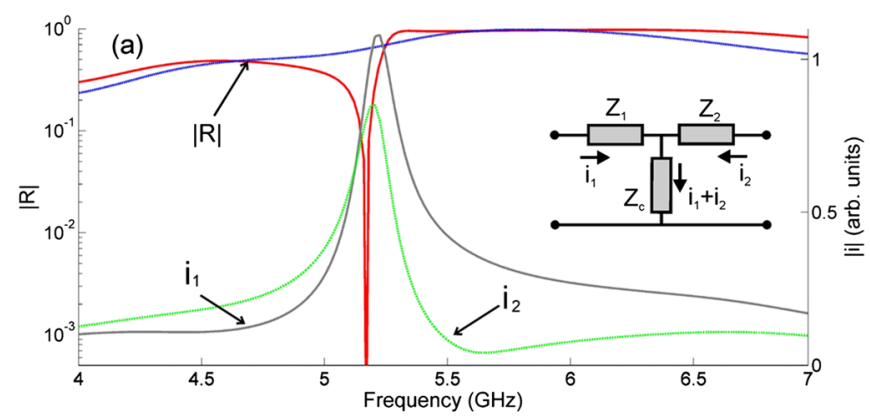

(b)

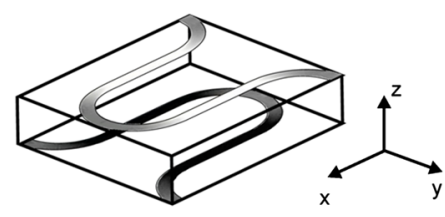

(c)
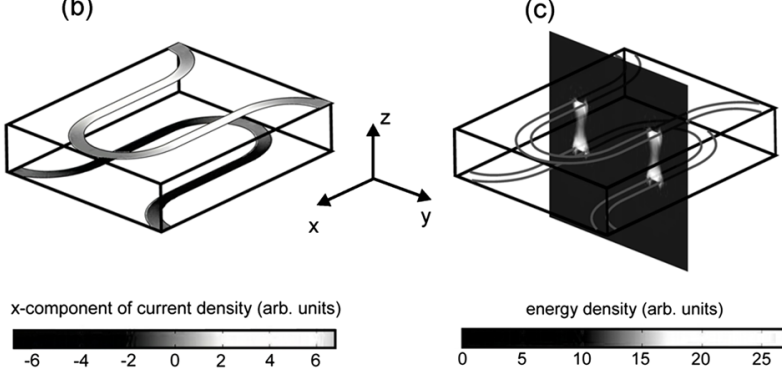

energy density (arb. units)

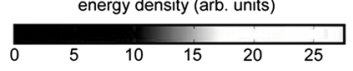

FIG. 3 (color online). (a) Representative reflection coefficient spectrum calculated using Eq. (1) for single layer (dotted blue) and two-layer (solid red) structures approximated by $Z_{0}=60+$ $18 \times 10^{-9}\left(\omega-\omega_{o}\right) i$ and $Z_{c}=55-60 i$, where $\omega_{o}=36.4 \times$ $10^{9} \mathrm{rad} / \mathrm{s}$. The gray and green curves represent the current strength on each layer. The equivalent circuit is shown in the inset. Numerically simulated current and energy density of the bilayered fish-scale at the resonant frequency $(5.5 \mathrm{GHz})$ are presented in (b) and (c), respectively.

Figs. 4(a) and 4(b), respectively. In both cases, stacking of multiple slabs results in an increase of maximum transmitted intensity and width of the normal dispersion frequency region. With each additional layer, a new

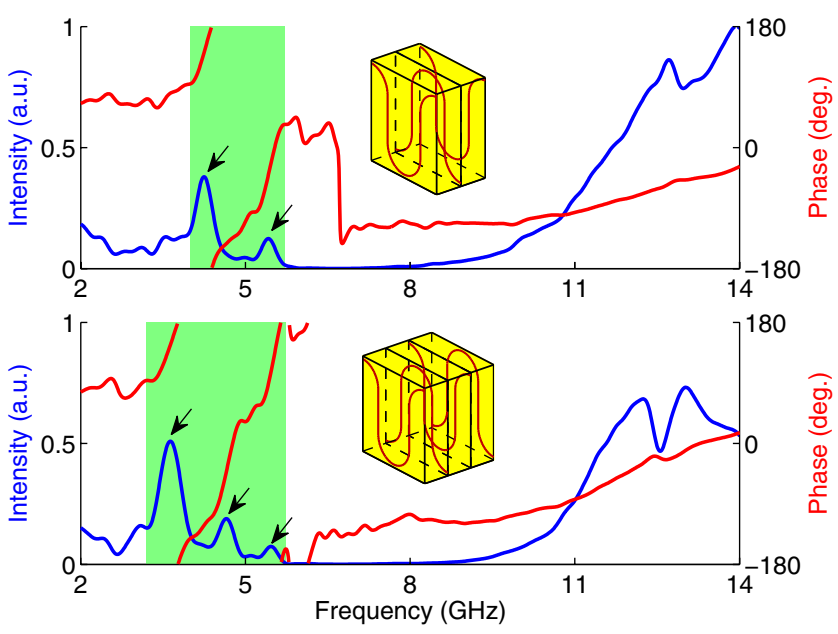

FIG. 4 (color online). Experimentally measured transmission amplitude (blue) and phase change (red) for three (a) and four (b) fish-scale layers. The arrows mark the positions of individual resonances. The area of normal dispersion is highlighted (green). The polarization is along the vertical axis as shown in the inset to Fig. 1. 
transmission peak appears shifted at lower frequencies with respect to the DFS case. At the same time, normal dispersion extends over a wide band exceeding 1.5 and $2.5 \mathrm{GHz}$ for three and four layers, respectively. This addresses the frequent bandwidth requirements of practical applications, whereas the thickness of the resulting metamaterial remains much smaller than the wavelength $(\lambda / 10)$. Although, further increase in the number of stacked layers is expected to result in additional transmission resonances and eventually in bulk, bandlike, behavior [37,38], the resulting medium will no longer be of subwavelength thickness (thus probably less suitable for highly integrated future photonic systems).

In conclusion, we demonstrated a metamaterial analogue of EIT media, where a virtually planar grating patterned on a subwavelength scale exhibits long optical delays of electromagnetic pulses without significant distortion and attenuation. This approach allows successive stacking of planar metamaterials, in order to increase the bandwidth of the effect. The resulting multilayered metamaterial structures are particularly appealing as broadband, ultracompact delay lines operating at prescribed wavelengths. At the stage of resubmission the authors also became aware of another work that introduced a similar concept in plasmonic metamaterials [39].

The authors would like to acknowledge the financial support of the Engineering and Physical Sciences Research Council, UK.

*N.Papasimakis@ soton.ac.uk

[1] L. Brillouin, Wave Propagation and Group Velocity (Academic Press, New York, 1960).

[2] C. G. B. Garrett and D. E. McCumber, Phys. Rev. A 1, 305 (1970).

[3] L. Casperson and A. Yariv, Phys. Rev. Lett. 26, 293 (1971).

[4] S. Chu and S. Wong, Phys. Rev. Lett. 48, 738 (1982).

[5] L. V. Hau, S.E. Harris, Z. Dutton, and C. H. Behroozi, Nature (London) 397, 594 (1999).

[6] A. V. Turukhin, V. S. Sudarshanam, M. S. Shahriar, J. A. Musser, B. S. Ham, and P. R. Hemmer, Phys. Rev. Lett. 88, 023602 (2001).

[7] M. S. Bigelow, N. N. Lepeshkin, and R. W. Boyd, Science 301, 200 (2003).

[8] M. Scalora, R. J. Flynn, S. B. Reinhardt, R. L. Fork, M. J. Bloemer, M. D. Tocci, C. M. Bowden, H.S. Ledbetter, J. M. Bendickson, J.P. Dowling, and R. P. Leavitt, Phys. Rev. E 54, R1078 (1996).

[9] M. Notomi, K. Yamada, A. Shinya, J. Takahashi, C. Takahashi, and I. Yokohama, Phys. Rev. Lett. 87, 253902 (2001).

[10] Y. A. Vlasov, M. O'Boyle, H. F. Hamann, and S. J. McNab, Nature (London) 438, 65 (2005).

[11] P. C. Ku, F. Sedgwick, C. J. Chang-Hasnain, P. Palinginis, T. Li, H. Wang, S. W. Chang, and S. L. Chuang, Opt. Lett. 29, 2291 (2004).
[12] Y. Okawachi, M. S. Bigelow, J. E. Sharping, Z. M. Zhu, A. Schweinsberg, D. J. Gauthier, R.W. Boyd, and A.L. Gaeta, Phys. Rev. Lett. 94, 153902 (2005).

[13] K. Y. Song, M. G. Herráez, and L. Thévenaz, Opt. Express 13, 82 (2005).

[14] J. T. Mok, C. M. de Sterke, I. C. M. Littler, and B.J. Eggleton, Nature Phys. 2, 775 (2006).

[15] M. Sandtke and L. Kuipers, Nat. Photon. 1, 573 (2007).

[16] K. L. Tsakmakidis, O. Hess, and A. D. Boardman, Nature (London) 450, 397 (2007).

[17] S. E. Harris, J. E. Field, and A. Imamoglu, Phys. Rev. Lett. 64, 1107 (1990).

[18] C. Liu, Z. Dutton, C. H. Behroozi, and L. V. Hau, Nature (London) 409, 490 (2001).

[19] S. E. Harris and L. V. Hau, Phys. Rev. Lett. 82, 4611 (1999).

[20] C. L. G. Alzar, M. A. G. Martinez, and P. Nussenzveig, Am. J. Phys. 70, 37 (2002).

[21] M.F. Yanik and S. Fan, Phys. Rev. Lett. 92, 083901 (2004).

[22] D. D. Smith, H. Chang, K. A. Fuller, A. T. Rosenberger, and R. W. Boyd, Phys. Rev. A 69, 063804 (2004).

[23] L. Maleki, A. B. Matsko, A. A. Savchenkov, and V.S. Ilchencko, Opt. Lett. 29, 626 (2004).

[24] M. F. Yanik, W. Suh, Z. Wang, and S. Fan, Phys. Rev. Lett. 93, 233903 (2004).

[25] A. Naweed, G. Farca, S. I. Shopova, and A. T. Rosenberger, Phys. Rev. A 71, 043804 (2005).

[26] Q. Xu, S. Sandhu, M. L. Povinelli, J. Shakya, S. Fan, and M. Lipson, Phys. Rev. Lett. 96, 123901 (2006).

[27] K. Totsuka, N. Kobayashi, and M. Tomita, Phys. Rev. Lett. 98, 213904 (2007).

[28] F. Xia, L. Sekaric, and Y. Vlasov, Nat. Photon. 1, 65 (2007).

[29] N. Papasimakis, V. A. Fedotov, S. L. Prosvirnin, and N. I. Zheludev, QELS (CLEO/QELS 2007), Baltimore, MD, USA, 6-11 May 2007.

[30] N. Papasimakis, V. A. Fedotov, S. L. Prosvirnin, and N. I. Zheludev, in Photonic Metamaterials: From Random to Periodic, OSA Technical Digest (CD) (OSA, Washington, DC, 2007), paper ThBPDP6.

[31] B. A. Munk and R. J. Luebbers, IEEE Trans. Antennas Propag. AP-22, 776 (1974).

[32] C. Antonopoulos, R. Cahill, E. A. Parker, and I. M. Sturland, Proc. IEE Microw. Ant. Propag. 144, 415 (1997).

[33] V. A. Fedotov, M. Rose, S. L. Prosvirnin, N. Papasimakis, and N. I. Zheludev, Phys. Rev. Lett. 99, 147401 (2007).

[34] V. A. Fedotov, P. L. Mladyonov, S. L. Prosvirnin, and N. I. Zheludev, Phys. Rev. E 72, 056613 (2005).

[35] Y. Q. Li and M. Xiao, Phys. Rev. A 51, 4959 (1995).

[36] M. Fleischhauer, A. Imamoglu, and J. P. Marangos, Rev. Mod. Phys. 77, 633 (2005).

[37] J. Valentine, S. Zhang, T. Zentgraf, E. Ulin-Avila, D. A. Genov, G. Bartal, and X. Zhang, Nature (London) 455, 376 (2008).

[38] N. Liu, L. Fu, S. Kaiser, H. Schweitzer, and H. Giessen, Adv. Mater. 20, 3859 (2008).

[39] S. Zhang, D. A. Genov, Y. Wang, M. Liu, and X. Zhang, Phys. Rev. Lett. 101, 047401 (2008). 